\title{
Lack of evidence to support the association of polymorphisms within the TNFSF4 gene and coronary heart disease in a Chinese Han population
}

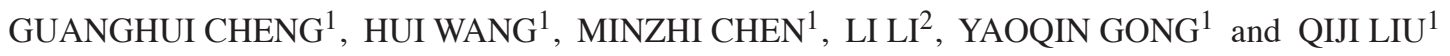 \\ ${ }^{1}$ Department of Medical Genetics and Key Laboratory for Experimental Teratology of the Ministry of Education, \\ Shandong University School of Medicine; ${ }^{2}$ Key Laboratory of Cardiovascular Remodeling and Function Research, \\ Chinese Ministry of Education and Chinese Ministry of Public Health, Department of Cardiology, Qilu Hospital, \\ Shandong University, Jinan 250012, P.R. China
}

Received September 8, 2010; Accepted December 6, 2010

DOI: $10.3892 / \mathrm{etm} .2010 .188$

\begin{abstract}
Coronary heart disease (CHD) is a complex disorder resulting from the interaction of a number of genetic and environmental factors. Increasing evidence has shown that OX40 ligand (OX40L), also known as tumor necrosis factor superfamily member 4 (TNFSF4), plays a key role in the pathogenesis of atherosclerosis. However, there have been inconsistent reports in various populations, and further studies are required to clarify this issue. A gene-based association study was conducted using five single-nucleotide polymorphisms (SNPs) reported in previous studies. The five SNPs (rs1234314, rs45454293, rs3850641, rs1234313 and rs3861950) were genotyped in 547 unrelated CHD patients and 601 healthy controls in a case-control study using polymerase chain reaction and restriction fragment length polymorphism. rs1234314, rs3850641 and rs3861950 were further genotyped in an additional 512 cases and 520 controls using the TaqMan SNP genotyping method. A possible relationship between the five SNPs and the severity of CHD was investigated. The results revealed no significant association between the TNFSF4 polymorphism and CHD. In addition, the stratified analysis of genotypic and allelic frequencies showed no association between the TNFSF4 polymorphism and CHD in either gender. Finally, no significant correlation between the TNFSF4 polymorphism and CHD severity was detected. These findings do not support a role of the TNFSF4 gene in CHD pathogenesis in the Chinese Han population.
\end{abstract}

Correspondence to: Dr Qiji Liu, Department of Medical Genetics and Key Laboratory for Experimental Teratology of the Ministry of Education, Shandong University School of Medicine, Jinan, Shandong 250012, P.R. China

E-mail: liuqiji@sdu.edu.cn

Key words: tumor necrosis factor super family member 4, coronary heart disease, polymorphism, association study

\section{Introduction}

Coronary heart disease (CHD) is the leading cause of death in industrialized countries, and its prevalence is rapidly increasing in China. Recent research has shown that inflammation plays a critical role in CHD pathogenesis and in other manifestations of atherosclerosis (1). Experimental evidence has confirmed that activated $\mathrm{T}$ cells are implicated in atherogenesis $(2,3)$.

Numerous studies have implicated TNFSF4/TNFRSF4 in the activation of $\mathrm{T}$ cells, as well as that of macrophages (4). TNFSF4, also known as OX40L, is primarily expressed by activated $\mathrm{B}$ cells, vessel endothelial cells, macrophages, dendritic cells and certain activated $\mathrm{T}$ cells. This generates costimulatory signals by interacting with TNFRSF4 on activated $\mathrm{T}$ lymphocytes and enhances the proliferation and differentiation of $\mathrm{T}$ lymphocytes and the development and survival of memory $\mathrm{T}$ cells (5). Thus, it is suggested that the TNFSF4-TNFRSF4 pathway plays a key role in atherosclerosis through its participation in $\mathrm{T}$ cell activation.

Numerous genetic studies have indicated a relationship between TNFSF4-TNFRSF4 and cardiovascular diseases. In 2006, Ria et al confirmed that genetic variation in TNFRSF4 was associated with myocardial infarction (MI) in a Swedish population (6). Furthermore, Mashimo et al found an association between TNFRSF4 gene polymorphisms and essential hypertension (7). There is current evidence showing that TNFSF4 is the gene underlying the atherosclerosis susceptibility locus 1 (Ath1) in mice, and that genetic polymorphisms in TNFSF4 are associated with MI and the severity of coronary artery disease (CAD) in humans (8). A subsequent study by Malarstig et al suggested that TNFSF4 variants are associated with the risk of incident atherothrombosis and venous thromboembolism in Caucasions (9). A recent study demonstrated that interruption of the TNFSF4-TNFRSF4 pathway attenuates atherogenesis in LDL receptor-deficient mice (10). However, despite the convincing evidence of disease association, a study involving a Chinese Han population is lacking. The present study aimed to replicate previous findings. We investigated the association between genetic 
Table I. Clinical characteristics of the coronary heart disease patients and the control subjects.

\begin{tabular}{|c|c|c|c|c|c|c|c|c|c|}
\hline \multirow[t]{2}{*}{ Parameter } & \multicolumn{3}{|c|}{ Total subjects } & \multicolumn{3}{|c|}{ Male } & \multicolumn{3}{|c|}{ Female } \\
\hline & Case & Control & P-value & Case & Control & $\mathrm{P}$-value & Case & Control & P-value \\
\hline No. & 1059 & 1121 & & 682 & 714 & & 377 & 407 & \\
\hline Age (years) & $60.35 \pm 10.42$ & $60.82 \pm 11.21$ & 0.132 & $60.52 \pm 10.21$ & $60.45 \pm 10.36$ & 0.299 & $60.02 \pm 10.45$ & $60.98 \pm 10.88$ & 0.421 \\
\hline BMI $\left(\mathrm{kg} / \mathrm{m}^{2}\right)$ & $26.16 \pm 5.26$ & $24.65 \pm 4.38$ & 0.013 & $26.12 \pm 4.13$ & $24.17 \pm 5.22$ & 0.032 & $25.92 \pm 7.30$ & $24.25 \pm 5.93$ & 0.029 \\
\hline SBP (mmHg) & $132.23 \pm 15.12$ & $121.21 \pm 9.45$ & 0.002 & $133.75 \pm 12.23$ & $120.23 \pm 9.56$ & 0.001 & $131.92 \pm 11.45$ & $120.10 \pm 10.20$ & 0.004 \\
\hline DBP $(\mathrm{mmHg})$ & $78.25 \pm 10.12$ & $75.45 \pm 9.72$ & 0.011 & $76.59 \pm 12.23$ & $75.12 \pm 7.52$ & 0.045 & $74.12 \pm 13.14$ & $73.35 \pm 9.65$ & 0.038 \\
\hline Glu (mmol/l) & $5.92 \pm 1.43$ & $5.19 \pm 0.89$ & 0.038 & $5.87 \pm 1.48$ & $5.45 \pm 0.78$ & 0.133 & $5.85 \pm 1.81$ & $5.02 \pm 1.19$ & 0.041 \\
\hline $\mathrm{TC}(\mathrm{mmol} / \mathrm{l})$ & $4.98 \pm 1.01$ & $4.75 \pm 0.63$ & 0.021 & $4.74 \pm 1.13$ & $4.69 \pm 0.83$ & 0.235 & $5.10 \pm 1.12$ & $4.86 \pm 0.29$ & 0.154 \\
\hline TG (mmol/l) & $1.97 \pm 1.23$ & $1.11 \pm 0.59$ & 0.004 & $1.93 \pm 0.87$ & $1.08 \pm 0.83$ & 0.021 & $2.08 \pm 1.01$ & $1.08 \pm 0.62$ & 0.001 \\
\hline
\end{tabular}

BMI, body mass index; SBP, systolic blood pressure; DBP, diastolic blood pressure; Glu, glucose; TC, total cholesterol; TG, triglyceride.

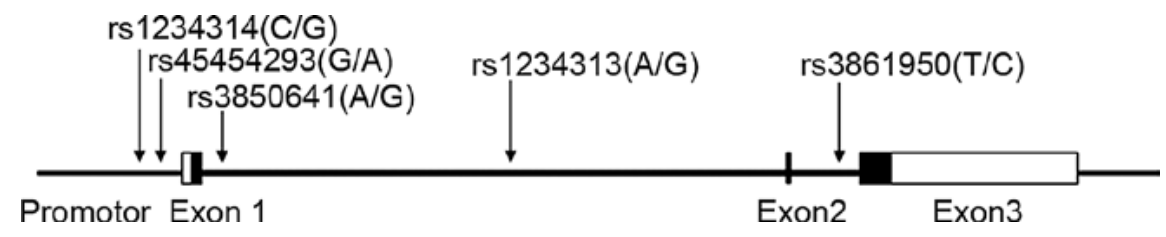

Figure 1. Positions of the SNPs in the TNFSF4 gene region genotyped in the present study.

variations in the TNFSF4 gene and CHD in a Chinese Han population.

\section{Materials and methods}

Study subjects. Subjects with Han Chinese ethnicity $(n=2180)$ were included in this case-control study. A total of 1059 patients with clinically manifested CHD, 682 males and 377 females, were recruited from Qilu Hospital of Shandong University between September 2006 and March 2010. The CHD patients were confirmed by coronary angiography to have $>50 \%$ stenosis in 1 or more coronary arteries. Patients who had a history of hyperthyroidism, secondary hypertension, chronic liver disease, chronic renal disease, acute infection and hematologic diseases were excluded from the study. A total of 1121 unrelated subjects (714 males and 407 females) were randomly selected as controls from a health check-up center in Qilu Hospital during the same period. The controls were free of CHD according to medical history and showed no clear ischemic changes by electrocardiography or symptoms of chest pain. Subjects with congestive heart failure, peripheral vascular disease, rheumatic heart disease, pulmonary heart disease, chronic kidney and hepatic disease were excluded.

A structured questionnaire based on interviews and clinical examinations was employed to characterize the patients and the controls. These included details of medical history, family history of CHD and other traditional risk factors of CHD, such as drug intake, cigarette smoking and alcohol consumption. Blood pressure, weight, height, waistline and hip circumference were also checked, and body mass index and waist-to-hip ratio were calculated. The clinical and demographic characteristics of the patients are shown in Table I. Genomic DNA was extracted from peripheral blood leukocytes by a standard salting-out method. This study was approved by the Ethics Committee of Shandong University School of Medicine, and informed consent was obtained from the participants.

Single-nucleotide polymorphism selection. The singlenucleotide polymorphisms (SNPs) examined here (rs3850641, rs1234313 and rs3861950) were as previously investigated in the two case-control samples from Sweden (8). The two other SNPs (rs45454293 and rs1234314) are located in the 5'-flanking region and may represent potential functional variants. One of them (rs45454293) was previously associated with incident venous thromboembolism (9). The locations of the SNPs are shown in Fig. 1.

Single-nucleotide polymorphism genotyping. Initially, the SNPs were genotyped using the PCR-RFLP method. SNPs rs3850641, rs1234314 and rs3861950 were genotyped using the TaqMan SNP genotyping method with assay-on-demand probes and primers (C_2955962_10 for rs1234314, C_26492316_10 for rs3850641 and C_8920839_10 for rs3861950; Applied Biosystems, Foster City, CA, USA) in the further recruited samples. For the PCR-RFLP, the gene sequences harboring the five sites were obtained from Genbank. Primers were designed with Primer Premier 5.0. The primers and restriction enzymes were: rs1234314 forward, 5'-tatc tgctgggtgcctcatg and reverse, 5'-gtcagacactgtgttagatg, ScrF I; rs45454293 forward, 5'-ttttagtggtaaagggtacctggtgtct and reverse, 5'-ataagctttgaagattatttcttctttgagct, Sac I; rs3850641 forward, 5'-tttgaagctttgagtcactgatatacetggtctaccaa 
and reverse, 5'-gcacgcacacattgctccgctatta, Mfe I; rs1234313 forward, 5'-cactgatgggcatttgggtt and reverse, 5'-ccactgcettgccatacct, Hpy CH4 IV; rs3861950 forward, 5'-caccetttgcccatagttc and reverse, 5'-ccttcagggagatgagataaa, Hin cII. PCR was carried out in a $10 \mu 1$ reaction volume containing $25 \mathrm{ng}$ of genomic DNA, 5 pmol of each primer, $100 \mu \mathrm{mmol} / 1$ of each dNTP, $2 \mu \mathrm{l}$ 5X PCR buffer and $1 \mathrm{U}$ Taq DNA polymerase. Amplification was carried out with an initial denaturation step at $94^{\circ} \mathrm{C}$ for $5 \mathrm{~min}$, followed by 35 cycles of denaturation at $94^{\circ} \mathrm{C}$ for $40 \mathrm{sec}$, annealing at a suitable temperature for $40 \mathrm{sec}$, extension at $72^{\circ} \mathrm{C}$ for $50 \mathrm{sec}$ with a final extension at $72^{\circ} \mathrm{C}$ for $10 \mathrm{~min}$. The PCR products containing the SNPs were digested with the suitable restriction enzyme (NEB, Beijing, China). The products were separated by electrophoresis on $1.5-2.5 \%$ agarose gel. The genotyping accuracy in the samples was confirmed by direct sequencing of the PCR products for certain randomly chosen samples.

Statistical analysis. Genotype frequencies of the SNPs detected were tested for Hardy-Weinberg equilibrium. Variations in genotype and allelic frequencies between case and control groups, odds ratios (OR), 95\% confidence intervals (CI), and logistic regression analysis were calculated using Plink 1.07. Continuous variables were displayed as the mean \pm standard deviation (SD), and the comparison of continuous variables was carried out using the Student's t-test. A p-value $<0.05$ was considered significant. Bonferroni correction was used to correct the multiple test. For the 5 SNPs each having 3 model tests included in the study, the adjusted $\mathrm{P}$ value significance threshold was $0.05 /(5 * 3)=0.003$. Power calculations were performed with the Power calculator for genetic association analysis by Menashe et al (11). For rs3850641, we referred to a study by Wang et al (8) in which the relative risk of rs3850641 in myocardial infarction was 1.4. For the other SNPs, the power was calculated under the assumption of a disease prevalence of $0.10, \mathrm{D}$-prime of 1 , dominant model and using the allelic frequency of each SNP as marker frequency.

\section{Results}

Clinical and demographic characteristics of the cases. The body mass index (BMI), systolic blood pressure (SBP), diastolic blood pressure (DBP), glucose (Glu), total cholesterol (TC) and triglyceride (TG) all demonstrated significant variations between the cases and controls. According to the examination results, $>85 \%$ of the cases had a history of MI. Compared with the controls, the cases had higher SBP, DBP, Glu, and TG levels. The clinical characteristics of the study subjects are shown in Table I.

Association of the polymorphisms with coronary heart disease. The distributions of all five SNPs were in Hardy-Weinberg equilibrium $(\mathrm{P}>0.05)$ in both the $\mathrm{CHD}$ and control groups. The distributions of genotypic and allelic frequencies of these SNPs in each group are shown in Tables II and III. Neither genotypes nor alleles differed between the case and control groups in the overall distribution (all $\mathrm{P}>0.05$ ). Meanwhile, we established the recessive, dominant models and Cochran-Armitage trend test using PLINK; no significant differences in the models were detected between the two groups (all $\mathrm{P}>0.05$ ).
Case-only association study. A case-only study was conducted to determine the effect of TNFSF4 variations on disease severity. The patients were divided into three subgroups according to the numbers of coronary arteries involved. The frequencies of the five SNPs in each group were compared. As shown in Table IV, there were no significant differences in the distribution of genotypic and allelic frequencies between each group. Although the p-value for rs3861950 between singlevessel and double-vessel, and single-vessel vs. triple-vessel was $<0.05$, the significance threshold was not reached after adjustment.

Power calculation. For rs3850641, our sample size had the power of $>90 \%$ when $\alpha$ was 0.05 under that assumption. For the other four SNPs, our sample size had $\geq 80 \%$ power to detect the effect of a relative risk of 1.4.

\section{Discussion}

Atherosclerosis is characterized by a complex multifactorial pathophysiology. It was formerly considered a disease of lipid accumulation, yet it actually involves an ongoing inflammatory response. Recent research has demonstrated that inflammation plays a pivotal role in the pathogenesis of atherosclerotic coronary artery disease. Vascular inflammation plays a predominant role in the initiation, progression and final steps of atherosclerosis (12). Numerous studies have shown that the TNFSF4-TNFRSF4 pathway plays a critical role in the pathogenesis of cardiovascular diseases.

We first investigated the association of the TNFSF4 gene and CHD using 547 patients and 601 controls. No significant differences were found in the genotypic and allelic frequency distribution between the two groups in the Chinese Han population studied. Moreover, even after adjusting for age, gender, BMI, SBP, DBP, Glu, TC and TG, no significant differences were found for the SNPs typed. To exclude falsenegative results due to the small sample size, we genotyped rs3850641, rs1234313 and rs3861950 in additional samples using the TaqMan assay method. In the Stockholm Coronary Atherosclerosis Risk Factor (SCARF) study, the minor allele of SNP rs3850641 in TNFSF4 was found to be associated with increased risk of MI in women (8). However, in our study, significant differences in genotypes were not found in either gender for the five SNPs, even after adjusting for age, gender, BMI, SBP, DBP, Glu, TC and TG.

In a previous study, the minor allele of rs3850641 in TNFSF4 was significantly more frequent in individuals with MI than in the controls in two independent human cohorts (8), while no significant difference was observed in our samples, and the frequency of the minor allele was even slightly higher in the controls. Also, no significant difference was found between the allelic frequencies in our data and the Han Chinese in Beijing (HCB) data from Hapmap. Analysis of the linkage disequilibrium (LD) structure using Haploview showed there is not much difference in the LD of the TNFSF4 gene between HCB and Utah residents of northern and western European ancestry.

A number of possibilities may account for the lack of association between the SNPs and CHD in this case-control study, including diagnostic heterogeneity, sample size, 


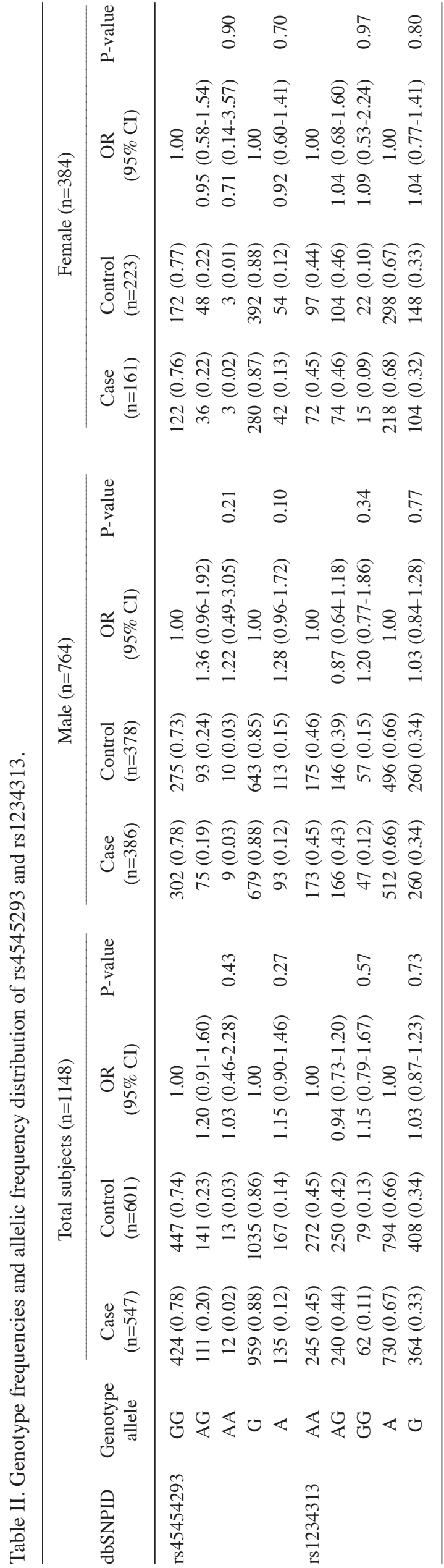

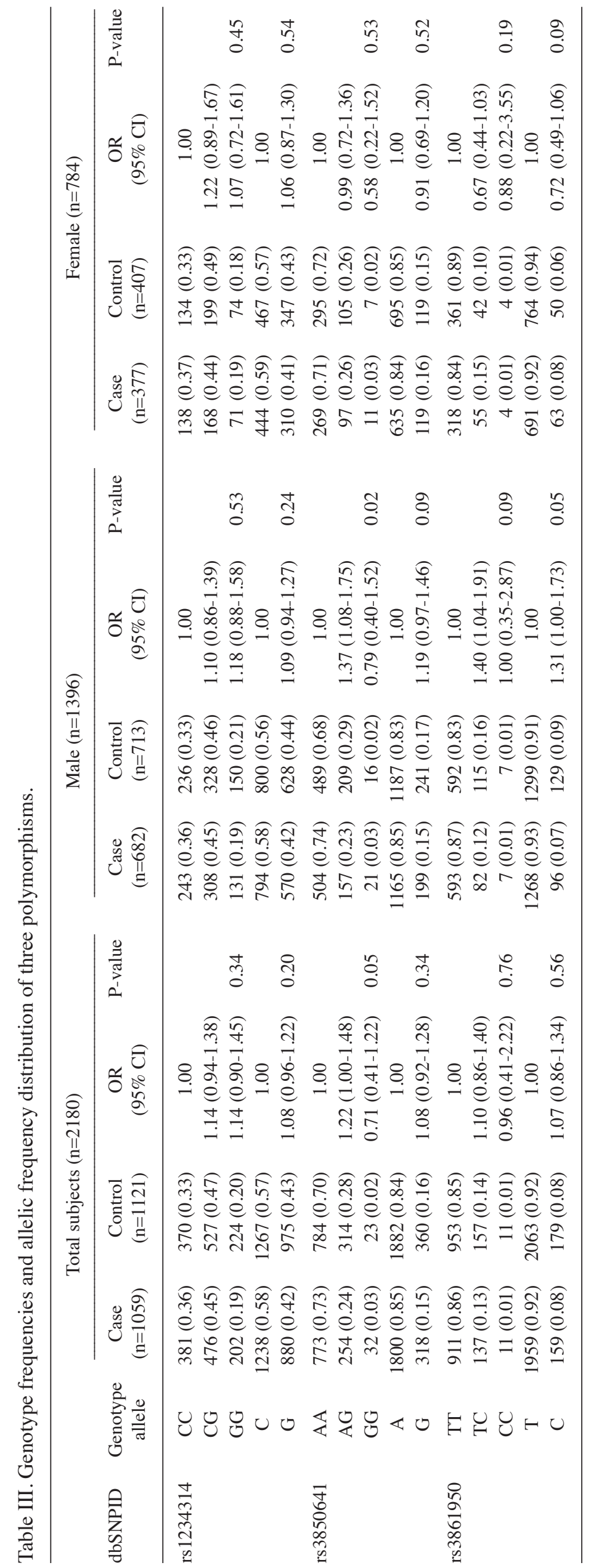


Table IV. Genotype frequencies and allelic frequencies in cases with a different number of coronary artery involved vessels.

\begin{tabular}{|c|c|c|c|c|c|c|c|c|c|c|}
\hline \multirow{4}{*}{$\begin{array}{l}\text { Single- } \\
\text { vessel } \\
(266)\end{array}$} & \multicolumn{2}{|c|}{ rs1234314 } & \multicolumn{2}{|c|}{ rs 45454293} & \multicolumn{2}{|c|}{ rs3850641 } & \multicolumn{2}{|c|}{ rs1234313 } & \multicolumn{2}{|c|}{ rs3861950 } \\
\hline & $\mathrm{CC}$ & $93(0.35)$ & GG & $109(0.78)$ & AA & $186(0.70)$ & $\mathrm{AA}$ & $55(0.40)$ & TT & $221(0.83)$ \\
\hline & CG & $125(0.47)$ & $\mathrm{AG}$ & $27(0.19)$ & $\mathrm{AG}$ & $69(0.26)$ & $\mathrm{AG}$ & $64(0.46)$ & $\mathrm{TC}$ & $40(0.15)$ \\
\hline & GG & $48(0.18)$ & $\mathrm{AA}$ & $3(0.03)$ & GG & $11(0.04)$ & GG & $20(0.14)$ & $\mathrm{CC}$ & $5(0.02)$ \\
\hline & $\mathrm{C}$ & $311(0.58)$ & G & $245(0.88)$ & A & $441(0.83)$ & A & $174(0.63)$ & $\mathrm{T}$ & $482(0.91)$ \\
\hline & G & $221(0.42)$ & $\mathrm{A}$ & $33(0.12)$ & $\mathrm{G}$ & $91(0.17)$ & $\mathrm{G}$ & $104(0.37)$ & $\mathrm{C}$ & $50(0.09)$ \\
\hline Double- & $\mathrm{CC}$ & $107(0.37)$ & GG & $114(0.78)$ & $\mathrm{AA}$ & $209(0.72)$ & $\mathrm{AA}$ & $73(0.50)$ & TT & $255(0.88)$ \\
\hline vessel & $\mathrm{CG}$ & $130(0.45)$ & $\mathrm{AG}$ & $29(0.20)$ & $\mathrm{AG}$ & $70(0.24)$ & $\mathrm{AG}$ & $58(0.40)$ & $\mathrm{TC}$ & $26(0.09)$ \\
\hline (290) & GG & $53(0.18)$ & $\mathrm{AA}$ & $3(0.02)$ & GG & $11(0.04)$ & GG & $15(0.10)$ & $\mathrm{CC}$ & $9(0.03)$ \\
\hline & $\mathrm{C}$ & $344(0.60)$ & G & $257(0.88)$ & $\mathrm{A}$ & $488(0.84)$ & A & $204(0.70)$ & $\mathrm{T}$ & $536(0.92)$ \\
\hline & G & $236(0.40)$ & A & $35(0.12)$ & $\mathrm{G}$ & $92(0.16)$ & $\mathrm{G}$ & $88(0.30)$ & $\mathrm{C}$ & $44(0.08)$ \\
\hline Triple- & $\mathrm{CC}$ & $185(0.37)$ & GG & $194(0.76)$ & $\mathrm{AA}$ & $371(0.74)$ & $\mathrm{AA}$ & $116(0.46)$ & TT & $436(0.87)$ \\
\hline vessel & $\mathrm{CG}$ & $220(0.44)$ & $\mathrm{AG}$ & $54(0.21)$ & $\mathrm{AG}$ & $115(0.23)$ & $\mathrm{AG}$ & $115(0.45)$ & $\mathrm{TC}$ & $60(0.12)$ \\
\hline$(501)$ & GG & $96(0.19)$ & $\mathrm{AA}$ & $6(0.03)$ & GG & $15(0.03)$ & GG & $23(0.09)$ & $\mathrm{CC}$ & $5(0.01)$ \\
\hline & $\mathrm{C}$ & $596(0.59)$ & G & $442(0.87)$ & $\mathrm{A}$ & $857(0.86)$ & A & $347(0.68)$ & $\mathrm{T}$ & $932(0.93)$ \\
\hline & $\mathrm{G}$ & $412(0.41)$ & A & $66(0.13)$ & G & $145(0.14)$ & G & $161(0.32)$ & $\mathrm{C}$ & $70(0.07)$ \\
\hline Control & $\mathrm{CC}$ & $370(0.33)$ & GG & $447(0.74)$ & $\mathrm{AA}$ & $784(0.70)$ & $\mathrm{AA}$ & $272(0.45)$ & TT & $953(0.85)$ \\
\hline (1121) & CG & $527(0.47)$ & $\mathrm{AG}$ & $141(0.23)$ & $\mathrm{AG}$ & $314(0.28)$ & $\mathrm{AG}$ & $250(0.42)$ & $\mathrm{TC}$ & $157(0.14)$ \\
\hline & GG & $224(0.20)$ & $\mathrm{AA}$ & $13(0.03)$ & GG & $23(0.02)$ & GG & $79(0.13)$ & $\mathrm{CC}$ & $11(0.01)$ \\
\hline & $\mathrm{C}$ & $1267(0.57)$ & G & $1035(0.86)$ & A & $1882(0.84)$ & A & $794(0.66)$ & $\mathrm{T}$ & $2063(0.92)$ \\
\hline & $\mathrm{G}$ & $975(0.43)$ & A & $167(0.14)$ & $\mathrm{G}$ & $360(0.16)$ & $\mathrm{G}$ & $408(0.34)$ & $\mathrm{C}$ & $179(0.08)$ \\
\hline S vs. C & & & & & & & & & & \\
\hline P-value & & 0.42 & & 0.37 & & 0.56 & & 0.27 & & 0.29 \\
\hline OR & & 1.08 & & 1.20 & & 0.93 & & 0.86 & & 0.84 \\
\hline $95 \% \mathrm{CI}$ & & $(0.89-1.31)$ & & $(0.80-1.78)$ & & $(0.72-1.19)$ & & $(0.66-1.13)$ & & $(0.60-1.16)$ \\
\hline D vs. C & & & & & & & & & & \\
\hline P-value & & 0.22 & & 0.39 & & 0.91 & & 0.22 & & 0.75 \\
\hline OR & & 1.12 & & 1.18 & & 1.01 & & 1.19 & & 1.06 \\
\hline $95 \% \mathrm{CI}$ & & $(0.93-1.35)$ & & $(0.80-1.75)$ & & $(0.79-1.30)$ & & $(0.90-1.57)$ & & $(0.75-1.49)$ \\
\hline T vs. C & & & & & & & & & & \\
\hline P-value & & 0.16 & & 0.62 & & 0.25 & & 0.37 & & 0.32 \\
\hline OR & & 1.11 & & 1.08 & & 1.13 & & 1.11 & & 1.16 \\
\hline $95 \% \mathrm{CI}$ & & $(0.96-1.29)$ & & $(0.80-1.47)$ & & $(0.92-1.39)$ & & (0.89-1.38) & & $(0.87-1.54)$ \\
\hline S vs. D & & & & & & & & & & \\
\hline $\mathrm{P}$-value & & 0.77 & & 0.99 & & 0.58 & & 0.07 & & 0.28 \\
\hline OR & & 0.97 & & 0.98 & & 0.91 & & 1.39 & & 0.79 \\
\hline $95 \%$ CI & & $(0.76-1.23)$ & & $(0.60-1.64)$ & & $(0.67-1.25)$ & & $(0.98-1.96)$ & & $(0.52-1.21)$ \\
\hline D vs. T & & & & & & & & & & \\
\hline P-value & & 0.94 & & 0.68 & & 0.46 & & 0.65 & & 0.66 \\
\hline OR & & 1.01 & & 0.91 & & 0.90 & & 1.08 & & 0.91 \\
\hline $95 \% \mathrm{CI}$ & & $(0.82-1.24)$ & & $(0.59-1.41)$ & & $(0.68-1.19)$ & & $(0.79-1.47)$ & & $(0.62-1.35)$ \\
\hline$S$ vs. T & & & & & & & & & & \\
\hline P-value & & 0.80 & & 0.65 & & 0.17 & & 0.59 & & 0.09 \\
\hline OR & & 0.97 & & 1.12 & & 0.82 & & 0.92 & & 0.72 \\
\hline $95 \% \mathrm{CI}$ & & $(0.79-1.20)$ & & $(0.71-1.73)$ & & $(0.62-1.09)$ & & $(0.67-1.26)$ & & $(0.50-1.06)$ \\
\hline
\end{tabular}

S, single-vessel; D, double-vessel; T, triple-vessel, C, control; All P-values are allelic P. The sample number of single-vessel, double-vessel, triple-vessel and controls for rs45454293 and rs 1234313 is $139,146,254$ and 601 , respectively.

population stratification and various genetic backgrounds in the various populations. To increase the homogeneity of our patients, we also performed a case-only association study; the genotypic and allelic frequencies did not indicate any trend in difference between the groups. With our sample size, we expected a power of at least $80 \%$ in detecting an effect of relative risk $\geq 1.4$ for each of the SNPs even at $\alpha=0.05$. Therefore, our failure to detect an association 
between CHD and these five SNPs may not have been due to the sample size.

Our results suggest that the SNPs studied in the TNFSF4 gene are unlikely to contribute to the CHD risk in the Chinese Han population. These results were consistent with those of Koch et al who did not find any association between the TNFSF4 gene and MI in a German population (13). In addition, Olofsson et al, in an association study of TNFSF4 gene variations with the risk for ischemic stroke using expression analysis, no significant association was found (14).

In conclusion, the present study aimed to determine whether the TNFSF4 gene is associated with CHD in a Chinese Han population. We did not find any significant association between five SNPs studied and CHD in our samples. Our results suggest that the TNFSF4 gene is unlikely to be a major susceptibility gene for CHD in our population.

\section{Acknowledgements}

This work was supported by grants of the National Natural Science Foundation of China (no. 30671956), the National Basic Research Program of China (973 Program) grant 2007CB512001 and the National High-Tech Research and Development Program of China; grant no. 2006AA02A406.

\section{References}

1. Hansson GK: Inflammation, atherosclerosis, and coronary artery disease. N Engl J Med 352: 1685-1695, 2005.

2. Hansson GK, Libby P, Schonbeck U and Yan ZQ: Innate and adaptive immunity in the pathogenesis of atherosclerosis. Circ Res 91: 281-291, 2002.

3. De Boer OJ, Becker AE and van der Wal AC: T lymphocytes in atherogenesis-functional aspects and antigenic repertoire. Cardiovasc Res 60: 78-86, 2003.
4. Walker LS, Gulbranson-Judge A, Flynn S, Brocker T and Lane PJ: Co-stimulation and selection for T-cell help for germinal centres: The role of CD28 and OX40. Immunol Today 21: 333-337, 2000

5. Weinberg AD: Ox40: targeted immunotherapy - implications for tempering autoimmunity and enhancing vaccines. Trends Immunol 23: 102-109, 2002.

6. Ria M, Eriksson P, Boquist S, Ericsson CG, Hamsten A and Lagercrantz J: Human genetic evidence that OX40 is implicated in myocardial infarction. Biochem Biophys Res Commun 339: 1001-1006, 2006.

7. Mashimo Y, Suzuki Y, Hatori K, Tabara Y, Miki T, Tokunaga K, Katsuya T, Ogihara T, Yamada M, Takahashi N, Makita Y, Nakayama T, Soma M, Hirawa N, Umemura S, Ohkubo T, Imai Y and Hata A: Association of TNFRSF4 gene polymorphisms with essential hypertension. J Hypertens 26: 902-913, 2008.

8. Wang X, Ria M, Kelmenson PM, Eriksson P, et al: Positional identification of TNFSF4, encoding OX40 ligand, as a gene that influences atherosclerosis susceptibility. Nat Genet 37: 365-372, 2005.

9. Malarstig A, Eriksson P, Rose L, Diehl KA, Hamsten A, Ridker PM and Zee RY: Genetic variants of tumor necrosis factor superfamily, member 4 (TNFSF4), and risk of incident atherothrombosis and venous thromboembolism. Clin Chem 54: 833-840, 2008.

10. Van Wanrooij EJ, van Puijvelde GH, de Vos P, Yagita $H$, van Berkel TJ and Kuiper J: Interruption of the TNFRSF4/ TNFSF4 (OX40/OX401) pathway attenuates atherogenesis in low-density lipoprotein receptor-deficient mice. Arterioscler Thromb Vasc Biol 27: 204-210, 2007.

11. Menashe I, Rosenberg PS and Chen BE: PGA: Power calculator for case-control genetic association analyses. BMC Genet 9: 36 , 2008.

12. Hansson GK, Robertson AK and Soderberg-Naucler C: Inflammation and atherosclerosis. Annu Rev Pathol 1: 297-329, 2006.

13. Koch W, Hoppmann P, Mueller JC, Schomig A and Kastrati A: Lack of support for association between common variation in TNFSF4 and myocardial infarction in a German population. Nat Genet 40: 1386-1388, 2008.

14. Olofsson PS, Soderstrom LA, Jern C, Sirsjo A, Ria M, Sundler E, de Faire U, Wiklund PG, Ohrvik J, Hedin U, Paulsson-Berne G, Hamsten A, Eriksson P and Hansson GK: Genetic variants of TNFSF4 and risk for carotid artery disease and stroke. J Mol Med 87: 337-346, 2009. 